\title{
Evaluación de los síntomas por déficit de macronutrientes en cinco especies de importancia agropecuaria de la región de Magallanes y Antártica Chilena
}

\author{
Evaluation of macronutrients deficiency symptoms in five species of \\ agricultural importance in Region of Magallanes and the Chilean Antarctic
}

Carlo Gorziglia ${ }^{1}$, Julio Yagello ${ }^{2} \&$ Sergio Radic $^{1}$

\section{Resumen}

Uno de los problemas agronómicos de la Región de Magallanes y Antártica Chilena es la deficiencia de macronutrientes en el suelo, ya sea por la naturaleza del suelo que puede ser pobre en el elemento en cuestión, o por no encontrarse disponible para la planta. Estas circunstancias afectan el correcto desarrollo de las especies que aquí se cultivan, es por eso que una herramienta importante para los agricultores es detectar en terreno cualquier posible anomalía nutricional mediante un diagnóstico visual temprano.

El estudio consistió en evaluar la deficiencia de macronutrientes (N-P-K), en cinco especies vegetales de importancia regional, tres especies de uso forrajero Medicago sativa L. (Leguminosae), Dactylis glomerata L. (Poaceae) y Trifolium repens L. (Fabaceae), y dos especies hortícolas Cucumis sativus L. (Curcubitaceae) y Rheum rhabarbarum L. (Polygonaceae), por medio de la técnica del elemento faltante. Se utilizaron contenedores pequeños donde se aplicó una solución nutritiva, en los cuales a cada uno de los nutrientes evaluados se le omite en un tratamiento, pero todos los demás nutrientes se aplican en niveles adecuados, además del tratamiento con la solución nutritiva completa. Las raíces de las especies en estudio se encontraban sumergidas en la solución mineral.

Hubo dos fases principales, la primera duró 21 días germinando las semillas en bandejas plásticas especialmente acondicionadas para este propósito, y en una segunda etapa las plántulas fueron repicadas a las jarras nutritivas para su establecimiento, en donde se mantuvieron con los distintos tratamientos por 41 días en un cultivo hidropónico. Las mediciones realizadas fueron el largo de raíz, altura de la parte aérea, producción de materia seca aérea y radical.

Como resultados se concluye que hay un menor desarrollovegetativo enlas especiesque se encuentran en la solución deficitaria de nitrógeno, seguido de la solución de fósforo y potasio respectivamente, a excepción de Rheum rhabarbarum L. y Trifolium repens L., en donde los tratamientos deficitarios de fósforo mostraron una altura y producción de materia seca igual o superior a los tratamientos sin potasio. Todas las especies hortícolas y forrajeras con tratamientos deficitarios de nitrógeno presentaron un menor desarrollo de altura. Cucumis sativus L. en el tratamiento sin nitrógeno fue la única especie estudiada que presentó una mayor producción de materia seca radical que el tratamiento sin fósforo. A partir de los 20 días se produce un aumento en el desarrollo aéreo de todos los tratamientos con soluciones no deficitarias, a excepción de Rheum rhabarbarum L. en donde el crecimiento tuvo un aumento lineal.

\section{Palabras clave:}

nitrógeno, fósforo, potasio, leguminosas, gramineas, especies hortícolas.

\footnotetext{
1 Departamento de Cs. Agropecuarias y Acuícolas Facultad de Ciencias Universidad de Magallanes Avenida Bulnes 01855, Punta Arenas, Chile $\measuredangle$ sergio.radic@umag.cl

2 Instituto de la Patagonia, Universidad de Magallanes
} 


\section{Abstract}

Soil macronutrient deficiencies are one of the agronomic problems present in the Region of Magallanes and the Chilean Antarctic either because the soils only contain very low concentrations of an element or it is not present in plant-available forms. These circumstances impede or slow growth and development of the crops produced in the region. Considering these challenges, it is important that farmers are able to visually detect signs of nutrient deficiencies early in the growing season.

This study evaluated the deficiency of macronutrients (N-P-K) in five species using the missing element technique. Plant species included Medicago sativa L. (Leguminosae), Dactylis glomerata L. (Poaceae), Trifolium repens L. (Fabaceae), Cucumis sativus L. (Curcubitaceae) and Rheum rhabarbarum L. (Polygonacea). Small containers were used to prepare nutrient solutions in which one of the three nutrients evaluated was omitted while maintaining adequate levels of the others. A treatment with a complete nutrient solution was also included. Plant roots were submerged in these mineral solutions.

Seeds were first planted into plastic trays and allowed to germinate. After 21 days, seedlings were transplanted into nutrition jars. Plants were grown under these conditions for 41 days. At the end of the growth stage, root and shoot length and radical and aerial dry matter weight were measured.

Vegetative development was most reduced in the nitrogen deficient treatment followed by phosphorus and then potassium, with the exception of $R$. rhabarbarum and $T$. repens. In these two species, plant height and dry matter weight in the phosphorus deficient treatment were equal to or greater than the potassium deficient treatment. Plant height for all species was most reduced by nitrogen deficiency. $C$. sativus in the nitrogen deficient treatment was the only species that presented greater dry matter root production compared to the phosphorus deficient treatment. After 20 days, there was an increase in shoot development in all species with the full nutrient solution except for $R$. rhabarbarum, in which growth was linear.

\section{Key words:}

nitrogen, phosphorus, potassium, legumes, grass, horticulture species.

\section{INTRODUCCIÓN}

La baja fertilidad de algunos nutrientes en los suelos de la Región de Magallanes y Antártica Chilena es una severa limitante en la producción de las praderas (Sáez, 1994). Uno de los primeros trabajos que se realizó para determinar deficiencias de nutrientes fue realizado por Schenkel et al. (1973), quienes señalaron que la baja productividad de los suelos de Magallanes incide sobre los rendimientos de muchas especies de vegetales relacionadas a los cultivos. Schenkel et al. (1973) encontraron que la intensidad de las deficiencias nutritivas decrece en el siguiente orden $\mathrm{P}>\mathrm{S}>\mathrm{K}>$ micronutrientes $>\mathrm{Ca}=\mathrm{Mg}$. La fertilización es una práctica que genera gran impacto en las praderas, al mejorar la producción de materia seca y la calidad nutritiva del forraje (Porta et al. 2006). Por otra parte, en el cultivo intensivo de hortalizas de Punta Arenas y Puerto Natales, la fertilización juega un papel importante y altamente rentable para lograr un producto de alta calidad y rendimiento (Sáez, 1994). Una cantidad insuficiente o excesiva de algún nutriente puede ocasionar problemas fisiológicos en el crecimiento y en la producción, para evitar esta situación se regula la fertilización (Razeto, 2009). Oliveira et al. (2006) señalan que una insuficiencia de nitrógeno da a lugar plantas poco desarrolladas con hojas pequeñas de color verde amarillento. Navarro (2003) agrega que el nitrógeno forma parte de todas las estructuras de las proteínas y de moléculas tan importantes como las purinas y pirimidinas, es componente de los ácidos nucléicos (ADN y ARN), de la clorofila y enzimas del grupo de los citocromos, indispensables para la fotosintesis y respiración. Según Covacevich \& Ruz (1996) el nitrógeno, presenta una baja disponibilidad en la región de Magallanes. En el caso del fósforo, éste presenta una baja disponibilidad en todos los suelos que no tienen un historial de fertilización a excepción de praderas habilitadas a partir de bosques (Sáez, 1994). En Medicago sativa L. la deficiencia de este elemento puede pasar desapercibida, pero luego ésta puede manifestarse con un color púrpura en tallos y hojas viejas debido a la acumulación de antocianinas (Ottman, 2010). El fósforo forma parte de las nucleoproteínas, lípidos y fosfolípidos. Además, desempeña un 
importante papel metabólico en la respiración y fotosíntesis (fosforilación), en el almacenamiento y transferencia de energía (NAD, NADP y ATP) y en la división y crecimiento celular (Bernal \& Espinosa, 2003). Para el caso del potasio en la región de Magallanes, se encuentra en niveles suficientes en el primer horizonte de suelo de las comunidades vegetales de vega, coironal y murtillar (Valle et al. 2015). La disponibilidad de potasio está directamente relacionada con el grado de lavado de bases de los suelos, vinculados a la precipitación y al material generador (Gutiérrez, 2003). Con frecuencia su deficiencia presenta puntos necróticos en las hojas. También se presenta una necrosis marginal que se inicia en las hojas viejas y luego las hojas se enrollan, la planta se torna débil y susceptible a rompimiento del tallo (Bernal \& Espinosa, 2003).

Las praderas sembradas en Magallanes ocupan una superficie de 50.000 ha, lo que representa el $0,9 \%$ de la superficie de uso ganadero, donde predominan especies exóticas introducidas, especialmente alfalfas y mezclas de gramíneas y trébol, que han sido fomentadas principalmente a través del Sistema de Incentivos para la Recuperación de Suelos Degradados (SIRSD), que impulsa el Ministerio de Agricultura (Bitsch, 2012). Medicago sativa L. es una planta de tallo ramificado y erecto, su sistema radical es muy grueso y alcanza algunos metros de profundidad (Dietl \& Fernández, 2009); es la principal siembra tradicional en la zona de estepa y de transición hacia el bosque descritas por Covacevich \& Ruz (1996). Trifolium repens L., es una especie de crecimiento rastrero con hojas de tallo largo, que se arrastran formando raíces en los nudos (Mcadam \& Olave, 2010). Covacevich (2006) señala que el trébol blanco es el pilar de la estrategia de mejoramiento de las praderas del sector húmedo de Magallanes. Corresponde a una especie de crecimiento postrado y posee estolones que le confieren una gran adaptación al pastoreo (Ganderast, 2001). Dactylis glomerata L., es una de las gramíneas forrajeras introducidas más importantes para Magallanes (SAG, 2003), esta especie se adapta a un amplio rango de situaciones y hoy en día se encuentra naturalizada en la región (Uribe, 2004). Por otra parte, una especie hortícola utilizada en la región es Rheum rhabarbarum L., que es una planta herbácea perenne miembro de la familia Polygonaceae, y presenta una parte subterránea que consiste en rizomas carnosos y un sistema radical fibroso, mientras que los pecíolos de las hojas se usan como alimento (Foust, 1991). Otra especie hortícola de importancia en la región es Cucumis sativus L., una hortaliza anual herbácea de tallos angulosos y trepadores, pertenece a la familia de las cucurbitáceas y posee un sistema radical muy extenso, el cual consta de una raíz principal pivotante que se ramifica para dar origen a numerosas raíces secundarias (Bolaños, 2001). En los últimos años ha pasado a ser una de las hortalizas más cultivadas en los invernaderos, siendo una de las fuentes de ingreso de la agricultura familiar campesina en la región, al ser uno de los productos más cotizados en el mercado (Tapia \& Pérez, 2016).

En relación a la sintomatología de deficiencias, el diagnóstico visual es rápido y barato, y además permite comparar los síntomas de deficiencia nutricional observados en un determinado cultivo, con los descritos en la literatura (Fontes, 2006). Aunque se debe considerar que la visualización de los síntomas, por sí sola, no es suficiente para hacer un diagnóstico definitivo del estado nutricional de la planta. Así, el diagnóstico visual junto al análisis químico de las hojas (órgano con mayor actividad metabólica, donde las variaciones en la nutrición de la planta pueden ser observadas con más facilidad), contribuyen a aumentar la seguridad en la evaluación del estado nutricional de las plantas (Römheld, 2012). Estas deficiencias son mejor visualizadas en los cultivos hidropónicos, donde las raíces de la planta son humedecidas en soluciones con nutrientes que poseen estos elementos (Resh, 2006). Según De Rijck y Schrevens (1998), las soluciones nutritivas son una mezcla acuosa de iones determinada por la proporción relativa de cationes, aniones y por la concentración total de iones. Texier (2015) agrega que algunas características importantes son el pH y la dureza.

De esta forma, el presente trabajo tuvo como objetivo la descripción de los sintomas de deficiencia de macronutrientes $(\mathrm{N}, \mathrm{P}, \mathrm{K})$ en cinco especies de importancia agropecuaria regional mediante el cultivo en soluciones nutritivas. 


\section{MATERIALES Y MÉTODOS}

El periodo de crecimiento de las 80 plantas tuvo una duración total de 62 días dividido en dos fases. La primera fase fue la de germinación y emergencia de las plántulas, donde las semillas fueron germinadas sobre un papel filtro saturado con agua destilada durante 21 días, y la segunda fase fue el establecimiento de las plantas durante 41 días con la aplicación de los tratamientos.

Se controló y registró las condiciones ambientales de la cámara de crecimiento donde se realizó el ensayo. La temperatura de la cámara se registró con un termómetro digital y fue regulada con un radiador de calefacción central ubicado en la pieza del experimento, presentando una temperatura promedio en el día de $22^{\circ} \mathrm{C}$ y en la noche de $18^{\circ} \mathrm{C}$. Se utilizó un fotoperiodo de 16 horas de luz y 8 de oscuridad. Las especies evaluadas fueron: alfalfa (Medicago sativa L. Ms), pasto ovillo (Dactylis glomerata L. - Dg), trébol blanco (Trifolium repens L. - Tr), pepino (Cucumis sativus L. - Cs) y ruibarbo (Rheum rhabarbarum L. - Rr). Se seleccionaron plántulas homogéneas en tamaño, que se replicaron a las jarras nutritivas, donde fue utilizada una planta

Tabla 1. Contenido en $\mathrm{mg} \mathrm{L}^{-1}$ de nutrientes presente en cada tratamiento a utilizar.

\begin{tabular}{cc}
\hline Nutriente & $\mathrm{mg} \mathrm{L}^{-1}$ \\
\hline $\mathrm{N}^{-N_{3}}$ & 230,03 \\
$\mathrm{~N}-\mathrm{NH}_{4}$ & 5,41 \\
$\mathrm{H}_{2} \mathrm{PO}_{4}(-)$ & 9,49 \\
$\mathrm{~K}$ & 29,32 \\
$\mathrm{Ca}$ & 55,31 \\
$\mathrm{~B}$ & 0,14 \\
$\mathrm{Na}$ & 1,97 \\
$\mathrm{Cl}$ & 3,04 \\
$\mathrm{Mo}$ & 1,00 \\
$\mathrm{Mg}$ & 6,56 \\
$\mathrm{Mn}$ & 0,30 \\
$\mathrm{Zn}$ & 0,07 \\
$\mathrm{Cu}$ & 0,002 \\
$\mathrm{Fe}$ & 1,00 \\
$\mathrm{SO}$ & 26,60 \\
\hline
\end{tabular}

por jarra. Cada jarra se pintó negra para evitar la entrada de luz a la solución nutritiva y se colocó una tapa de poliestireno expandido, con un agujero en el centro, por donde se introdujo el tallo de las plantas, estas fueron fijadas con una esponja de poliuretano de modo que la planta quedó con sus raíces suspendidas y sumergidas en la solución. En esta etapa se utilizó una adaptación de la solución nutritiva (Tabla 1) propuesta por Rengel y Robinson (1989). Las soluciones nutritivas fueron renovadas cada 48 horas, utilizando un volumen aproximado de $250 \mathrm{~mL}$ por jarra. Diariamente se oxigenó la solución mediante el trasvasije repetido de la misma solución. El agua destilada utilizada para la preparación de la solución poseía un $\mathrm{pH}$ de 5,2. La temperatura de la solución nutritiva en el día fue de $20^{\circ} \mathrm{C}$ y en la noche de $16^{\circ} \mathrm{C}$.

Los parámetros evaluados fueron: altura de plantas (se extrajo la planta y se estiró en una superficie, donde se realizó la medición desde el cuello de la planta hasta la punta más alta de la hoja más larga), largo de raíz, y producción de materia seca aérea y radical. Las mediciones de altura en el tiempo se realizaron con la planta en la jarra nutritiva y se midió desde la base de la planta inserta en la esponja de poliuretano a la hoja más alta. Las muestras se secaron en un horno SHEL LAB SL, donde se dejaron a $60^{\circ} \mathrm{C}$ por 48 horas (Vaieretti et al. 2007).

El ensayo tuvo un diseño experimental de bloques completos al azar, con dos bloques y dos tratamientos, que fueron especies y nutriente adicionado. Las soluciones nutritivas fueron cuatro, completo (N-P-K), sin N, sin Py sin K, para cada una de las cinco especies evaluadas. Cada tratamiento tuvo cuatro repeticiones, las cuales se ordenaron en dos repeticiones por bloque. El análisis estadístico se realizó mediante un análisis de varianza de dos vías para encontrar diferencias significativas entre soluciones nutritivas aplicadas para la misma especie. Cuando hubo diferencias estadísticas, se utilizó el LSD como test de comparaciones múltiples para encontrar diferencias entre medias, utilizando una significancia de $p<0,05$.

\section{RESULTADOS}

Como se muestra en la Tabla 2, para la altura de la parte aérea en Ms, existen diferencias 
estadísticas significativas entre todos los tratamientos. En $D g$ los tratamientos completo y sin potasio se diferenciaron estadísticamente de los tratamientos sin fósforo y sin nitrógeno. $\mathrm{Tr}$ en altura de la parte aérea presentó diferencias estadísticas significativas, entre el tratamiento completo y el resto de los tratamientos. En esta especie el tratamiento sin nitrógeno presentó diferencias en altura aérea con el tratamiento sin fósforo y el tratamiento completo, pero no existió una diferencia estadística significativa con el tratamiento sin potasio. En $R r$, el tratamiento sin nitrógeno no permitió el establecimiento de la planta en ninguno de los cuatro bloques. Mientras que el tratamiento sin fósforo no presentó diferencias estadísticas significativas entre los tratamientos sin $\mathrm{K}$ y completo. En el Cs existieron diferencias estadísticas significativas para el parámetro de altura aérea entre la mayoría de los tratamientos, excepto entre los tratamientos sin fósforo y sin nitrógeno. Mientras que para largo y materia seca radical, existieron diferencias estadísticas entre todos los tratamientos.

$\mathrm{Al}$ analizar el parámetro de largo de raíz en todas las especies (Tabla 2), se pudo mostrar que para $M s$ se presentaron diferencias significativas entre el tratamiento completo y los tratamientos sin fósforo y sin nitrógeno, mientras que no se presentaron diferencias estadísticas entre el tratamiento $\sin \mathrm{K}$ y el completo. En $\mathrm{Dg}$ el tratamiento completo y el tratamiento sin potasio no presentaron diferencias significativas entre ellos, pero sí hubo diferencias cuando los comparamos con los tratamientos sin fósforo y sin nitrógeno. En $\operatorname{Tr}$ solo se presentaron diferencias estadísticas significativas entre el tratamiento completo y el resto de los tratamientos en estudio, pero no entre los tratamientos deficitarios. En $R r$ se presentaron diferencias estadísticas significativas entre todos los tratamientos, al igual que en el caso del Cs, donde el tratamiento sin nitrógeno fue el que presentó menor largo radical, seguido del sin fósforo y posteriormente el sin potasio.

En cuanto a materia seca aérea (Tabla 3), en $M s$ se presentaron diferencias estadísticas significativas entre todos los tratamientos. En $D g$, Tr y Cs el tratamiento completo presentó diferencias estadísticas significativas con el resto de los tratamientos en estudio. En $R r$ no se presentó una diferencia estadística significativa entre el tratamiento completo y el sin fósforo, pero sí el tratamiento completo presentó diferencias con el tratamiento sin potasio y el sin nitrógeno. En cuanto a materia seca radical, en $M s$ se presentaron diferencias estadísticas significativas entre todos los tratamientos. En $D g$ el tratamiento completo presentó diferencias estadísticas significativas con el resto de los tratamientos en estudio, mientras que el tratamiento sin potasio no presentó diferencias con el tratamiento sin fósforo. En el Tr los tratamientos deficitarios no presentaron

Tabla 2. Altura aérea y largo de raíz promedio ( \pm D. E.) de cinco especies utilizadas en la región de Magallanes.

Nota: Medicago sativa L. - Ms; Dactylis glomerata L. - Dg; Trifolium repens L. - Tr; Cucumis sativus L. - Cs; Rheum rhabarbarum L. - Rr. Diferentes letras minúsculas indican diferencias significativas entre tratamientos para cada especie evaluada.

\begin{tabular}{|c|c|c|c|c|c|c|c|c|c|c|}
\hline \multirow{2}{*}{ Tratamientos } & \multicolumn{9}{|c|}{ Altura aérea $\left(\mathrm{cm} \mathrm{jarra}^{-1}\right)$} & \\
\hline & Ms & & $D g$ & & $\operatorname{Tr}$ & & $R r$ & & Cs & \\
\hline $\operatorname{Sin} N$ & $3,7 \pm 0,2$ & $\mathrm{~d}$ & $7,1 \pm 0,8$ & $\mathrm{~b}$ & $2,5 \pm 0,1$ & c & $0,1 \pm 0,1$ & $\mathrm{c}$ & $8,9 \pm 0,6$ & c \\
\hline $\operatorname{Sin} P$ & $6,0 \pm 0,5$ & c & $8,0 \pm 3,3$ & $\mathrm{~b}$ & $3,3 \pm 0,2$ & $\mathrm{~b}$ & $7,4 \pm 1,5$ & $\mathrm{ab}$ & $9,7 \pm 0,6$ & c \\
\hline $\operatorname{Sin} K$ & $8,1 \pm 0,7$ & $\mathrm{~b}$ & $11,6 \pm 1,1$ & $\mathrm{a}$ & $2,7 \pm 0,2$ & $\mathrm{bc}$ & $6,7 \pm 0,4$ & $\mathrm{~b}$ & $10,7 \pm 0,6$ & $\mathrm{~b}$ \\
\hline Completo & $11,1 \pm 1,9$ & $\mathrm{a}$ & $12,6 \pm 1,6$ & $\mathrm{a}$ & $4,7 \pm 0,6$ & $\mathrm{a}$ & $9,6 \pm 0,5$ & $\mathrm{ab}$ & $14,5 \pm 1,1$ & $\mathrm{a}$ \\
\hline \multicolumn{11}{|c|}{ Largo radical (cm jarra $\left.{ }^{-1}\right)$} \\
\hline $\operatorname{Sin} N$ & $3,0 \pm 0,2$ & c & $3,5 \pm 1,0$ & $\mathrm{~b}$ & $1,4 \pm 0,05$ & $\mathrm{~b}$ & $0,1 \pm 0,1$ & d & $12 \pm 1,8$ & d \\
\hline $\operatorname{Sin} P$ & $4,2 \pm 0,2$ & $\mathrm{~b}$ & $2,8 \pm 0,4$ & $\mathrm{~b}$ & $1,5 \pm 0,1$ & $\mathrm{~b}$ & $7,1 \pm 0,2$ & c & $5,4 \pm 1,1$ & c \\
\hline $\operatorname{Sin} \mathrm{K}$ & $8,8 \pm 0,5$ & a & $5,3 \pm 0,6$ & a & $1,6 \pm 0,1$ & $\mathrm{~b}$ & $8,6 \pm 0,7$ & $\mathrm{~b}$ & $9,2 \pm 0,6$ & $\mathrm{~b}$ \\
\hline Completo & $9,5 \pm 1,0$ & a & $6,4 \pm 0,5$ & a & $3,0 \pm 0,2$ & $\mathrm{a}$ & $9,8 \pm 0,5$ & $\mathrm{a}$ & $14 \pm 2,0$ & a \\
\hline
\end{tabular}


Tabla 3. Producción de materia seca promedio ( \pm D.E.)

de la parte aérea y radical de cinco especies utilizadas en la región de Magallanes.

\begin{tabular}{|c|c|c|c|c|c|c|c|c|c|c|}
\hline \multirow[t]{2}{*}{ Tratamientos } & \multicolumn{10}{|c|}{ Materia seca parte aérea (g MS jarra-1) } \\
\hline & Ms & & $\mathrm{Dg}$ & & $\operatorname{Tr}$ & & $\operatorname{Rr}$ & & Cs & \\
\hline $\operatorname{Sin} N$ & $2,7 \pm 0,1$ & $d$ & $1,2 \pm 0,1$ & $\mathrm{~b}$ & $1,4 \pm 0,1$ & $\mathrm{~b}$ & $0,1 \pm 0,1$ & c & $33,4 \pm 5,6$ & $\mathrm{~b}$ \\
\hline $\operatorname{Sin} P$ & $3,5 \pm 0,4$ & c & $1,2 \pm 0,1$ & $\mathrm{~b}$ & $1,5 \pm 0,09$ & $\mathrm{~b}$ & $12,3 \pm 0,5$ & $\mathrm{ab}$ & $33,8 \pm 3,9$ & $\mathrm{~b}$ \\
\hline $\operatorname{Sin} \mathrm{K}$ & $5,4 \pm 2,4$ & $\mathrm{~b}$ & $1,2 \pm 0,09$ & $\mathrm{~b}$ & $1,5 \pm 0,09$ & $\mathrm{~b}$ & $11,0 \pm 0,5$ & $\mathrm{~b}$ & $36,2 \pm 1,7$ & $\mathrm{~b}$ \\
\hline \multirow[t]{2}{*}{ Completo } & $5,0 \pm 0,2$ & $\mathrm{a}$ & $1,8 \pm 0,09$ & a & $2,6 \pm 0,2$ & $\mathrm{a}$ & $14,0 \pm 1,4$ & $\mathrm{a}$ & $44,7 \pm 2,9$ & $\mathrm{a}$ \\
\hline & \multicolumn{10}{|c|}{ Materia seca radical (g MS jarra-1 ${ }^{-1}$ ) } \\
\hline $\operatorname{Sin} N$ & $1,6 \pm 0,1$ & $\mathrm{~d}$ & $0,2 \pm 0,05$ & c & $0,3 \pm 0,1$ & $\mathrm{~b}$ & $0,1 \pm 0,1$ & $\mathrm{~b}$ & $8,0 \pm 0,9$ & $\mathrm{~b}$ \\
\hline Sin $P$ & $1,9 \pm 0,2$ & c & $0,3 \pm 0,08$ & bc & $0,3 \pm 0,05$ & $\mathrm{~b}$ & $4,4 \pm 0,2$ & $\mathrm{a}$ & $4,6 \pm 0,5$ & c \\
\hline $\operatorname{Sin} K$ & $2,7 \pm 0,2$ & $\mathrm{~b}$ & $0,3 \pm 0,05$ & $\mathrm{~b}$ & $0,4 \pm 0,05$ & $\mathrm{ab}$ & $4,6 \pm 0,5$ & $\mathrm{a}$ & $8,0 \pm 1,2$ & $\mathrm{~b}$ \\
\hline Completo & $3,1 \pm 0,1$ & $\mathrm{a}$ & $0,5 \pm 0,05$ & a & $0,6 \pm 0,1$ & $\mathrm{a}$ & $5,2 \pm 0,6$ & $\mathrm{a}$ & $12,0 \pm 1,9$ & $\mathrm{a}$ \\
\hline
\end{tabular}

Nota: Medicago sativa L. - Ms; Dactylis glomerata L. - Dg; Trifolium repens L. - Tr; Cucumis sativus L. - Cs; Rheum rhabarbarum L. - Rr. Diferentes letras minúsculas indican diferencias significativas entre tratamientos para cada especie evaluada.

diferencias significativas entre sí, mientras que el tratamiento completo no presentó diferencias estadísticas significativas con el tratamiento sin potasio, pero sí con los tratamientos sin fósforo y sin nitrógeno. En $R r$ no hubo diferencias significativas entre el tratamiento completo, el sin fósforo y el sin potasio, mientras que éstos presentaron diferencias con el tratamiento deficitario de nitrógeno. En Cs el tratamiento completo presentó diferencias estadísticas significativas con el resto de los tratamientos, el tratamiento sin nitrógeno tuvo diferencias con el tratamiento sin fósforo, no presentando diferencias con el tratamiento sin potasio.

En la Fig. 1 se presenta la altura promedio de las distintas especies evaluadas, donde se puede ver que los tratamientos completos en $\mathrm{Tr}$, Ms, Cs y Rr presentaron diferencias estadísticas significativas con todo el resto de los tratamientos que presentaban deficiencia de algún nutriente. En el caso de Dg el tratamiento completo no presentó diferencia estadística significativa con el tratamiento sin potasio, pero sí con los demás tratamientos con deficiencias. El tratamiento sin nitrógeno para todas las especies presentó las menores alturas. Se puede ver que desde el día 20, se produce un punto de inflexión y en la mayoría de las especies con las soluciones completas, se comenzaron a diferenciar estadísticamente de las plantas de las soluciones deficitarias.

\section{DISCUSIÓN}

Kelling (2000), en su estudio sobre fertilización en Medicago sativa L., señala que los síntomas de déficit de nitrógeno comienzan con un color verde claro, sobre todo en las hojas inferiores y las plantas presentan un reducido desarrollo. Lo anterior concuerda con los resultados obtenidos en las características morfológicas (Fig. 2). Además, las plantas deficitarias en nitrógeno presentaron un $47 \%$ menos de materia seca total y un $66 \%$ menos altura que las con la solución completa.

En Medicago sativa L., la carencia de fósforo mostró que la producción de materia seca total fue un $34 \%$ menor y las plantas fueron $46 \%$ más bajas (Tabla 3) comparadas con las plantas que contenían la solución completa, aunque éstas no mostraron síntomas visuales claros de deficiencia en hojas, lo que concuerda con lo señalado por Ottman (2010) en su trabajo sobre síntomas de déficit en Medicago sativa L., donde señala que aunque la planta reduce su crecimiento, en un principio la deficiencia puede pasar desapercibida, y que luego ésta se manifiesta con un color púrpura en tallos y hojas viejas debido a la acumulación de antocianinas (Fig. 2).

El tratamiento sin potasio fue el que menor diferencia mostró con el tratamiento completo, esto puede deberse a que esta deficiencia no 

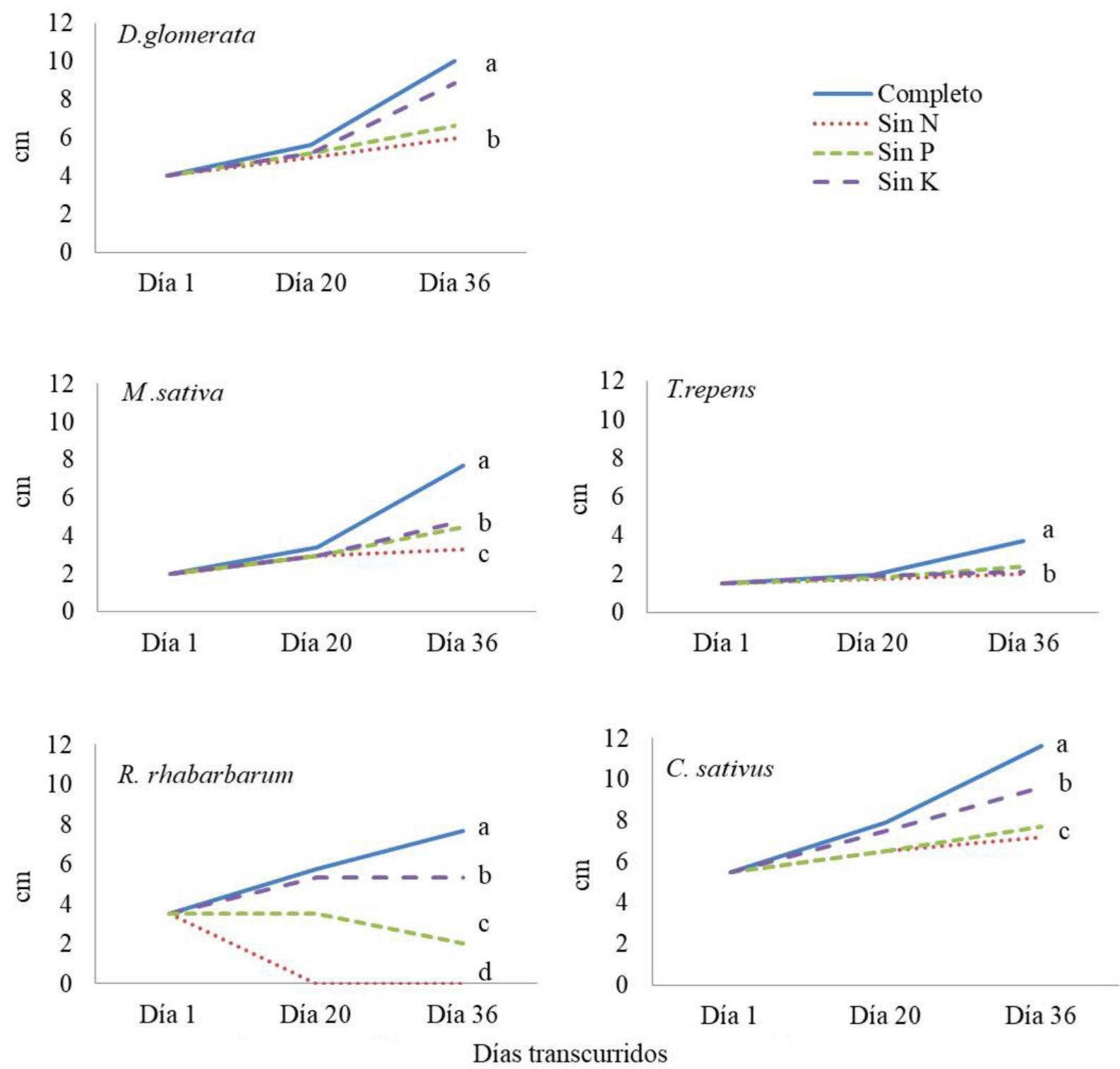

Fig. 1. Registro semanal de desarrollo aéreo, indicando las curvas de crecimiento promedio para altura de planta de cada una de las especies evaluadas por tratamiento.

presenta sintomas inicialmente, pero se puede ver reducido su crecimiento como indica Kumar y Kumar (2013). Por otro lado, el potasio es uno de los elementos nutritivos que mayor requerimiento tiene en alfalfa (Rodríguez, 2001), lo que podría generar un impacto inmediato en su desarrollo.

Trifolium repens L. presentó pequeños puntos blancos en los bordes de las hojas inferiores (Fig. 3) lo que concuerda con los resultados obtenidos por Archie y Vartha (1985), que describen la deficiencia de $\mathrm{K}$ con la aparición de patrones de pequeñas manchas blancas cerca de los márgenes de las hojas.

Salinas y Sanz (1981) estudiaron el déficit de macronutrientes con la técnica del elemento faltante en una especie de leguminosa, Pueraria phaseoloides y en tres de gramíneas, Adropogon gayanus, Brachiaria spp. y Panicum maximim. 

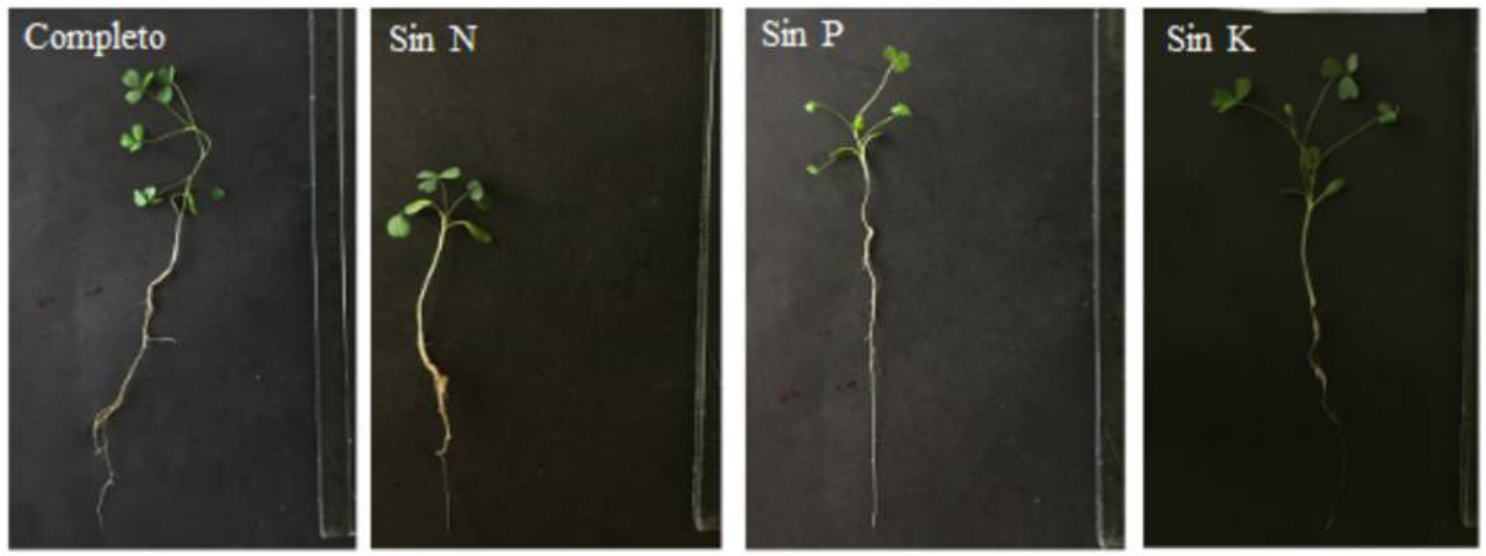

Fig. 2. M. sativa en los distintos tratamientos.
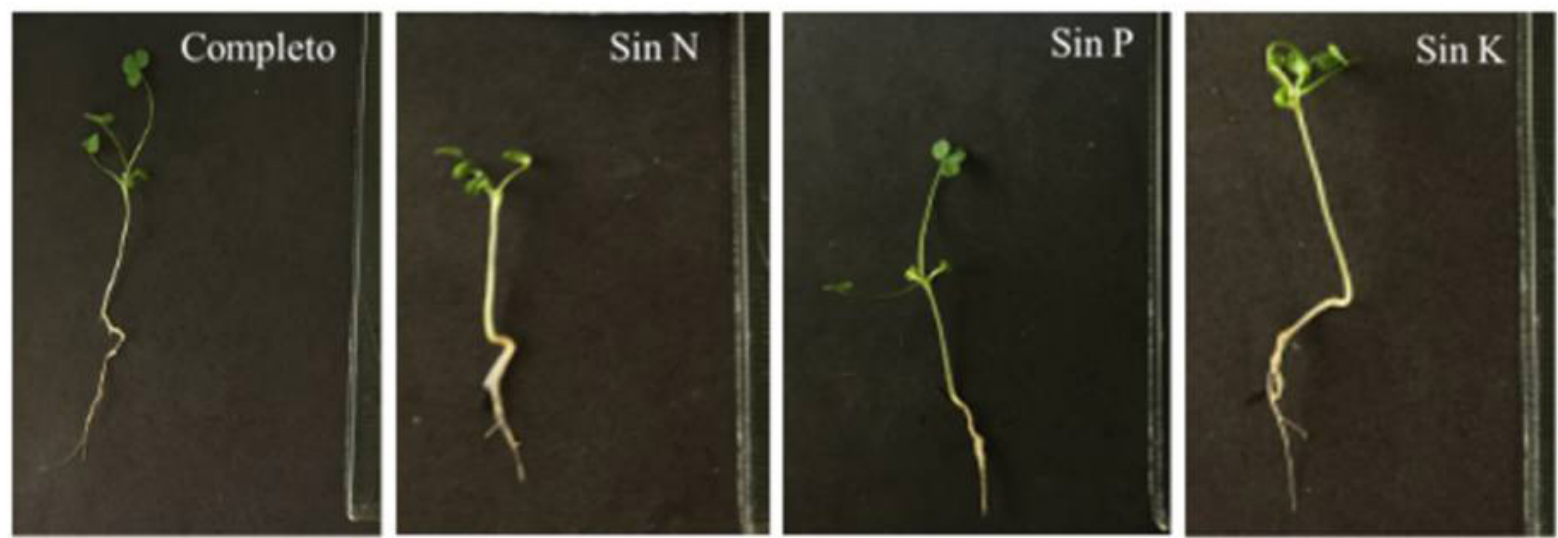

Fig. 3. T. repens en los distintos tratamientos.

Hay que señalar que las plantas de leguminosa no fueron inoculadas, al igual que en nuestro estudio, pero los primeros 20 días se regaron con solución completa, después de ese periodo pasaron a los tratamientos deficitarios. Los efectos de déficit de $\mathrm{N}, \mathrm{P}, \mathrm{K}$ entre estas dos leguminosas y una gramínea fueron bastante similares, lo que concuerda con los resultados obtenidos.

Los autores Zhang et al. (2012) señalan en su trabajo sobre deficiencias de macronutrientes en la etapa temprana de Cucumis sativus L. que el menor promedio de materia seca aérea se obtuvo con el tratamiento deficitario de nitrógeno, seguido del tratamiento de fósforo y potasio. El experimento anterior concuerda con los resultados obtenidos en este estudio en donde el menor promedio de materia seca aérea fue el tratamiento sin nitrógeno (33,48 g), seguido del sin fósforo $(33,80 \mathrm{~g})$ y sin potasio $(36,28 \mathrm{~g})$, el tratamiento completo presentó un peso de 44,78 g. Por otro lado, Zhang et al. (2012) muestran que el tratamiento que presentó mayor peso de materia seca radical fue el carente de fósforo, seguido del tratamiento completo, sin potasio y sin nitrógeno lo cual difiere de los resultados presentados en la Tabla 3; puesto que los mejores resultados se obtuvieron en el tratamiento completo $(12,98$ $\mathrm{g})$, seguido de potasio (8,03 g), fósforo (4,63 g) y nitrógeno $(8,00 \mathrm{~g})$ respectivamente. Esto pudo deberse a que el autor sometió las plantas a riegos con solución completa durante los primeros 21 días del ensayo.

Las raíces de Rheum rhabarbarum L. presentaron en todos los tratamientos a excepción del tratamiento sin potasio una coloración negro azabache (Fig. 4). Esto puede deberse a la falta de oxigenación en las raíces. Como exponen Gislerod y Kempton (1983), la carencia o escasez de oxígeno en la zona radicular de la planta se manifiesta con un color pardo oscuro, tal vez este 

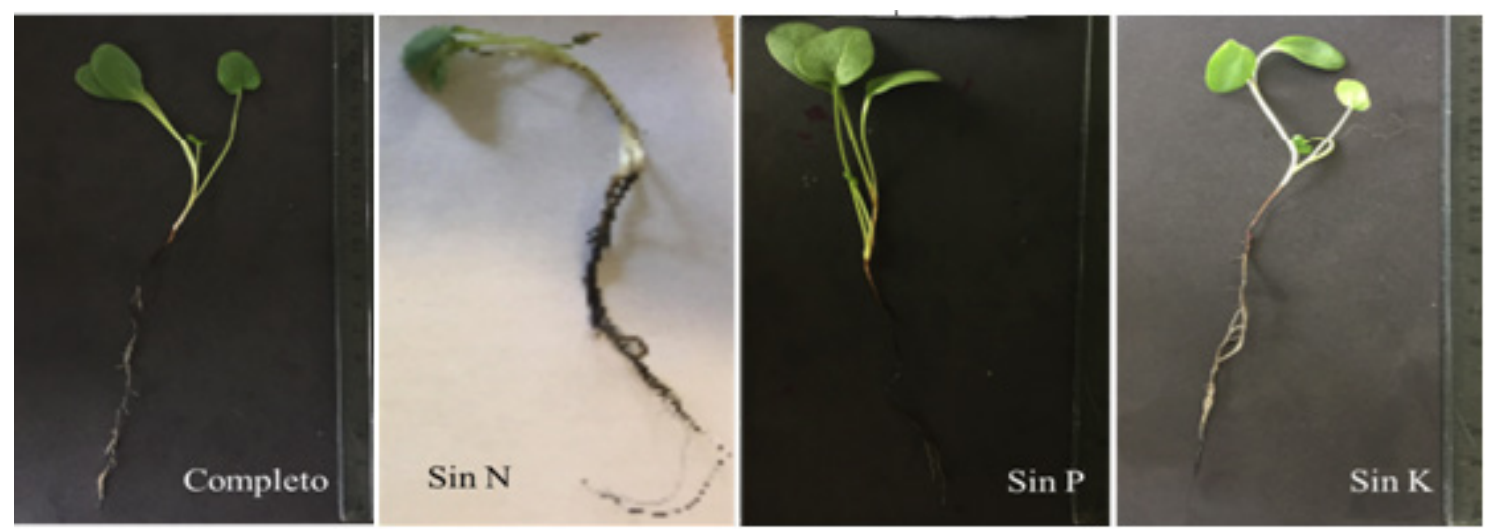

Fig. 4. R. rhabarbarum en los distintos tratamientos.

sea el síntoma más precoz y fácilmente detectable de los primeros problemas al respecto. Por otra parte, otros autores como Howard et al. (1994) indican que Phytophthora corona o "podredumbre de la raíz" es una enfermedad grave en el ruibarbo, generalmente produce lesiones leves y hundidas en la base de los tallos, que se agrandan rápidamente, dando como resultado hojas marchitas del colapso de todo el tallo. La corona y las raíces se vuelven marrones o negras y comienzan a desintegrarse. La muerte de los individuos cultivados bajo carencia de nitrógeno puede verse explicada por lo que señala Ingratta (1979), ya que el ruibarbo tiene un alto requerimiento de nitrógeno y recomienda la fertilización nitrogenada previa a la germinación.

Fernandes et al. (2005), en un estudio sobre aspectos morfológicos de Cyclanthera pedata (cucurbitáceas, nativa de Brasil), exponen que el tratamiento deficitario de nitrógeno presentó un $56,25 \%$ menos de materia seca radical que el tratamiento completo, similar a los resultados obtenidos en este estudio en donde las plantas con carencia de nitrógeno presentaron un 39\% menos de materia seca radical (Tabla 3) comparado con el tratamiento completo.

El efecto visual de déficit por N-P-Ken las hojas de Cucumis sativus L. se presentó primeramente en las hojas con carencia de nitrógeno, las hojas basales fueron pequeñas y de un color verde más claro que el de las hojas superiores. En las plantas con déficit de potasio las hojas basales mostraron una ligera clorosis marginal y pérdida de turgencia. Esto concuerda con lo que expone Zamora (2006), en su trabajo sobre deficiencias de Cucumis sativus L., donde señala que la deficiencia de fósforo se parece en un principio a la de nitrógeno, pero la hoja inmediatamente superior queda de un color verde oscuro.

En general los resultados de este estudio no difieren de los encontrados en otras especies, como por ejemplo en el de Riascos et al. (2009) en tomate, donde la materia seca aérea presentó menores resultados con el tratamiento sin nitrógeno seguido de sin fósforo y sin potasio. Al evaluar la producción de materia seca aérea en este estudio, se mostró que para Medicago sativa L. y Rheum rhabarbarum L., el tratamiento sin $\mathrm{N}$ fue el más determinante en su desarrollo, mientras que para Dactylis glomerata L. y Trifolium repens L. este nutriente fue más determinante en la parte radical. Aunque para Dactylis glomerata la deficiencia de fósforo también generó disminución en el desarrollo de largo de raíz, lo cual señalan Salinas y Sanz (1981), quienes indican que la deficiencia de P afecta directamente el metabolismo de la planta, a consecuencia de lo cual hay una reducción de la tasa de crecimiento.

$\mathrm{Si}$ bien es sabido que el N, P y K, limitan el crecimiento y desarrollo de los cultivos, en condiciones de campo, los mecanismos precisos para evaluar los síntomas de déficit por los cuales ocurren estas limitaciones son complejos y variables según la especie, etapa de desarrollo y condición del medio ambiente. Hay que tener en cuenta que los síntomas aparecen luego que el suministro es muy bajo y que cuando aparece la deficiencia puede ser tarde para corregirla, porque como podemos ver en los resultados de este estudio el desarrollo radicular y aéreo se ve afectado mucho antes de la aparición de sintomas claros en las hojas y raíces. 


\section{BIBLIOGRAFÍA}

Archie, W., \& Yartha, E. (1985). Performance of grasses on cultivated hill country in North Canterbury. New Zealand Journal of Experimental Agriculture, 13, 1-4.

Bernal, J., \& Espinosa, J. (2003). Manual de Nutrición y Fertilización de Pastos. Instituto de la Potasa y el Fósforo. Quito: INPOFOS.

Bitsch,M.(2012). Estrategiapara lacompetitividad del sector agroalimentario y forestal 20122020. Ministerio de Agricultura. Región de Magallanes y Antártica Chilena.

Bolaños, A. (2001). Introducción a la olericultura. San José, CR. EUNED. Costa Rica.

Covacevich, N., \& Ruz, J. (1996). Praderas en la zona austral: XII Región (Magallanes). En I. Ruiz (Ed.). Praderas para Chile. $2^{\mathrm{a}}$ ed. (pp. 639-655). Santiago de Chile: Ministerio de Agricultura, Instituto de Investigaciones Agropecuarias (INIA).

Covacevich, N. (2006). Trébol para Magallanes. Edición Especial, Tierra Adentro. Instituto de Investigaciones Agropecuarias. Punta Arenas, Chile.

De Rijck, G., \& Schrevens, E. (1998). Cationic Speciation in Nutrient Solutions as a Function of $\mathrm{pH}$. Journal of Plant Nutrition, 5(21), 861-870.

Dietl, W., Fernández, F., \& Venegas, C. (2009). Manejo Sostenible de Praderas: Su flora y vegetación. Boletín INIA Nº187. Instituto de Investigaciones Agropecuarias, Cauquenes, Chile.

Fernandes, L., Alves, D., Ramos, S., Oliveira, A., \& Martins, E. (2005). Nutrição mineral de plantas de maxixe-do-reino. Pesquisa Agropecuária Brasileira, 40, 719-722.

Fontes, P. (2006). Diagnóstico do estado nutricional das plantas. Vic, osa, MG: Universidade Federal de Vic, osa.

Foust, C. (1991). Culinary Rhubarb Production in North America: History and Recent Statistics. HortScience, 26(11), 13601363.

Ganderast, F. (2001). Antecedentes sobre la producción de praderas en Aysén. Instituto de Investigaciones Agropecuarias (INIA).
Centro regional de investigación Tamal Aike. Boletín INIA $\mathrm{N}^{\circ} 69$, Instituto de Investigaciones Agropecuarias, Coihaique, Chile.

Gislerod, H., \& Kemptom, R. (1983). The oxygen content of flowing nutrient solutions used for cucumber and tomato culture. Scientia Horticulturae, 20, 23-33.

Gutiérrez, T. (2003). Factores de la producción agrícola. Santiago de Chile: Universidad Tecnológica Metropolitana.

Howard, R., Garland, A., \& Seaman, L. (1994). Diseases and Pests of Vegetable Crops in Canada. The Canadian Phytopathological Society and the Entomological Society of Canada. Ottawa, M.O.M. Printing Ltd.

Ingratta, F. (1979). Growing Rhubarb. OMAF Factsheet. Vineland: Editorial Ontario.

Kelling, K. (2000). Alfalfa fertilization. Publ. A2448. University of Wisconsin. Wisconsin. Cooperative Extension Publishing.

Kumar, M., \& Kumar, S. (2013). Nutrient Deficiencies of Field Crops: Guide to Diagnosis and Management. Guide to Diagnosis and Management. Department of Agriculture, India. 400 p.

Mcadam, J., \& Olave R. (2010). Falkland Islands: Pasture Plant Guide. Department of Agriculture (DoA) and the Agri-Food and Biosciences Institute (AFBI). Belfast: AgriFood and Biosciences Institute.

Navarro, G. (2003). Química Agrícola: El suelo y los elementos químicos esenciales para la vida vegetal. Madrid: Mundi Prensa.

Oliveira, J., Afif, E., \& Mayor, M. (2006). Análisis de suelo y plantas y recomendaciones de abonados. Oviedo: Ediciones Universidad de Oviedo.

Ottman, M. (2010). Suboptimal Irrigation Strategies for Alfalfa in the Lower Colorado Region. Forage \& Grain Report, College of Agriculture and Life Sciences. Arizona: University of Arizona.

Porta, M., Hack, C., \& Tomei, C. (2006). El método del cultivo intensivo en macetas para el estudio de la fertilidad de suelos. Instituto Agrotécnico "Pedro M. Fuentes Godo". Calaceite, España. Facultad de Ciencias Agrarias, UNNE. 
Razeto, B. 2009. Nutrient deficiencies: Iron. (cap.1, pp.56-72). En: Symptoms of nutrient imbalances in fruit trees. Santiago, Chile: SQM. 189.

Rengel, Z., \& Robinson, D. (1989). Aluminium effects on growth and macronutrient uptake by annual ryegrass. Agronomy Journal, 81, 208 - 215.

Resh, H. (2006). Cultivos hidropónicos, 5ta edición. Madrid: Editorial Mundi-Prensa.

Riascos, J., Yaluzan, L., \& Unigarro, A. (2009). Sintomas de deficiencia de elementos mayores y secundarios en tomates de árbol bajo condiciones controladas. Revista de Ciencias Agrícolas, 1, 33-48.

Rodríguez, A. (2001). Soluciones Nutritivas en Hidroponía. Manual Práctico de Hidroponía. ( $3^{\text {a }}$ Ed.). Lima, Perú: Univerisdad Nacional Agraria la Molina.

Römheld, V. (2012). Diagnosis of deficiency and toxicity of nutrients En P. Marschner (Ed.), Marschner's mineral nutrition of higher plants (pp. 299-312). London: Academic Press.

Sáez, C. (1994). Caracterización de la fertilidad de los Suelos de la Región de Magallanes. Punta Arenas: Ediciones Universidad de Magallanes.

Salinas, J., \& Sanz, J. (1981). Sintomas de deficiencia de macronutrimentos $y$ nutrimentos secundarios en Pastos Tropicales. Guía de estudio. Centro Internacional de Agricultura Tropical. Cali, Colombia: CIAT.

Schenkel, G., Baher Le, P., Floody, H., \& Gajardo, M. (1973). Exploración de deficiencias nutritivas con suelos en macetas. XVII. Comportamiento de algunas fórmulas de fertilización, provincia de Magallanes, Continente. Agricultura Técnica, 34(3),
116-136.

Servicio Agrícola y Ganadero (SAG). (2003). El pastizal de Tierra del Fuego. Guía de uso condición actual y propuesta de seguimiento para determinación de tendencia. Servicio Agrícola y Ganadero Departamento de Protección de los Recursos Naturales Renovables. Gobierno Regional de Magallanes y Antártica Chilena. Ms.

Tapia, F., \& Pérez, C. (2016). Bases para la producción y comercialización de hortalizas en Magallanes. Boletín INIA ( $\left.\mathrm{N}^{\circ} 333\right)$. Instituto de Investigaciones Agropecuarias. Centro Regional de Investigación Kampenaike. Punta Arenas, Chile.

Texier, W. (2015). Hidroponía para todos. París: Editorial Mama Publishing.

Uribe, I. (2004). Manual de terreno para la identificación de especies en pastizales de la XII región. Punta Arenas: SAG XII Región de Magallanes.

Vaieretti, V., Díaz S., Vile D., \& Garnier E. (2007). Two Measurement Methods of Leaf Dry Matter Content Produce Similar Results in a Broad Range of Species. Annals of Botany, 99(5), 955-958.

Valle, S., Radic, S., \& Casanova, M. (2015). Suelos asociados a tres comunidades vegetales de pastoreo importantes en Patagonia Sur. Revista Agro Sur, 43(2), 89-99.

Zamora, E. (2016). Deficiencias nutricionales en cultivos protegidos. Ciudad de México: Universidad de Sonora.

Zhang, B., Chen, Q., Yang, Q., \& Liu, K. (2012). Effects of NPK deficiencies on root architecture and growth of cucumber. International Journal of Agriculture and Biology, 14, 145-148. 